\title{
Myometrial myxoidosis: A rare case seen in a patient of primary subfertility
}

\author{
Nilam Subedi1 ${ }^{1}$ MD; Padam Raj Pant ${ }^{1,2}$, PhD; Asmita Ghimire ${ }^{1}$, MD \\ ${ }^{1}$ Department of Gynecology \& Obstetrics, Grande International Hospital, Kathmandu, Nepal \\ ${ }^{2}$ Department of Gynecology \& Obstetrics, Institute of Medicine, Tribhuvan University Teaching Hospital, \\ Kathmandu, Nepal
}

Corresponding author

Nilam Subedi, MD

email: nsnilam16@gmail.com

Received 29 Oct 2018

Accepted 14 Dec 2018

\begin{abstract}
Myxoid mesenchymal lesions of the uterus are generally restricted to tumors. Myometrial myxoidosis is a rare benign tumor of the uterus. We report a case of myometrial myxoidosis diagnosed postoperatively with histopathological report in a 27 -year-old female with primary subfertility who had undergone myomectomy and adenomyomectomy. On gross examination the tumor was $13.5 \times 8 \times 2.5 \mathrm{~cm}$ in size. Microscopically, it showed interlacing bundles of smooth muscles intersecting each other. Sections from separate tissue showed muscle fascicles which were separated by abundant extracellular mucin producing a striking myxoid appearance (myxoidosis).

Only few cases of myometrial myxoidosis have been reported in literature. Of them, some were associated with lupus erythematosus and some with neurofibromatosis.

Keywords: Myxoidosis, myxoid, infertility
\end{abstract}

\section{Introduction}

Myxoid mesenchymal lesions occurring within the uterus are uncommon. Myometrial myxoidosis with secondary myometrial hypertrophy was reported in 2 patients with systemic lupus erythematosus. McCluggage et al. in another 3 patients reported it as an unusual pseudoneoplastic condition of uncertain etiology ${ }^{1}$.

\section{Case report}

A 27-year-old nulligravida married for 5 years came with complaint of inability to conceive for her duration of marriage. She also gave history of mass in lower abdomen, increased frequency of micturition and per vaginal spotting for 15 days. Thyroid function test was normal. On examination her vitals were within normal limits, there was no signs of pallor, icterus, cyanosis, edema, or dehydration. Per abdomen there was 24 weeks gestational size mass freely mobile, with regular margin, smooth surface and was firm in consistency. Her ultrasonogram abdomen showed intramural myoma measuring $12 \times 8 \mathrm{~cm}$ in posterior myometrial wall.

She was admitted, all investigations were done and planned for myomectomy. Intraoperative findings were: uterus was approximately of 24-weeks gestational size $(24 \times 18 \mathrm{~cm}), 10 \times 10 \mathrm{~cm}$ intramural myoma, myometrial wall thickened, adenomyoma was present. On chromopertubation, there was no spillage of dye on both sides.

\section{Discussion}

Patient in this case report was having primary subfertility with leiomyoma uterus. The main pathologic finding apart from leiomyoma was muscle fascicles which were separated by abundant extracellular mucin producing a striking myxoid appearance "myxoidosis".

Myxoid change in the uterus is very rare in absence of neoplasia. Veras et al. $^{2}$ reported 2 cases of myometrial myxoidosis in patients of lupus erythematosus and Pugh et al. ${ }^{3}$ reported 2 cases of uterine myxoid lesion in association with 


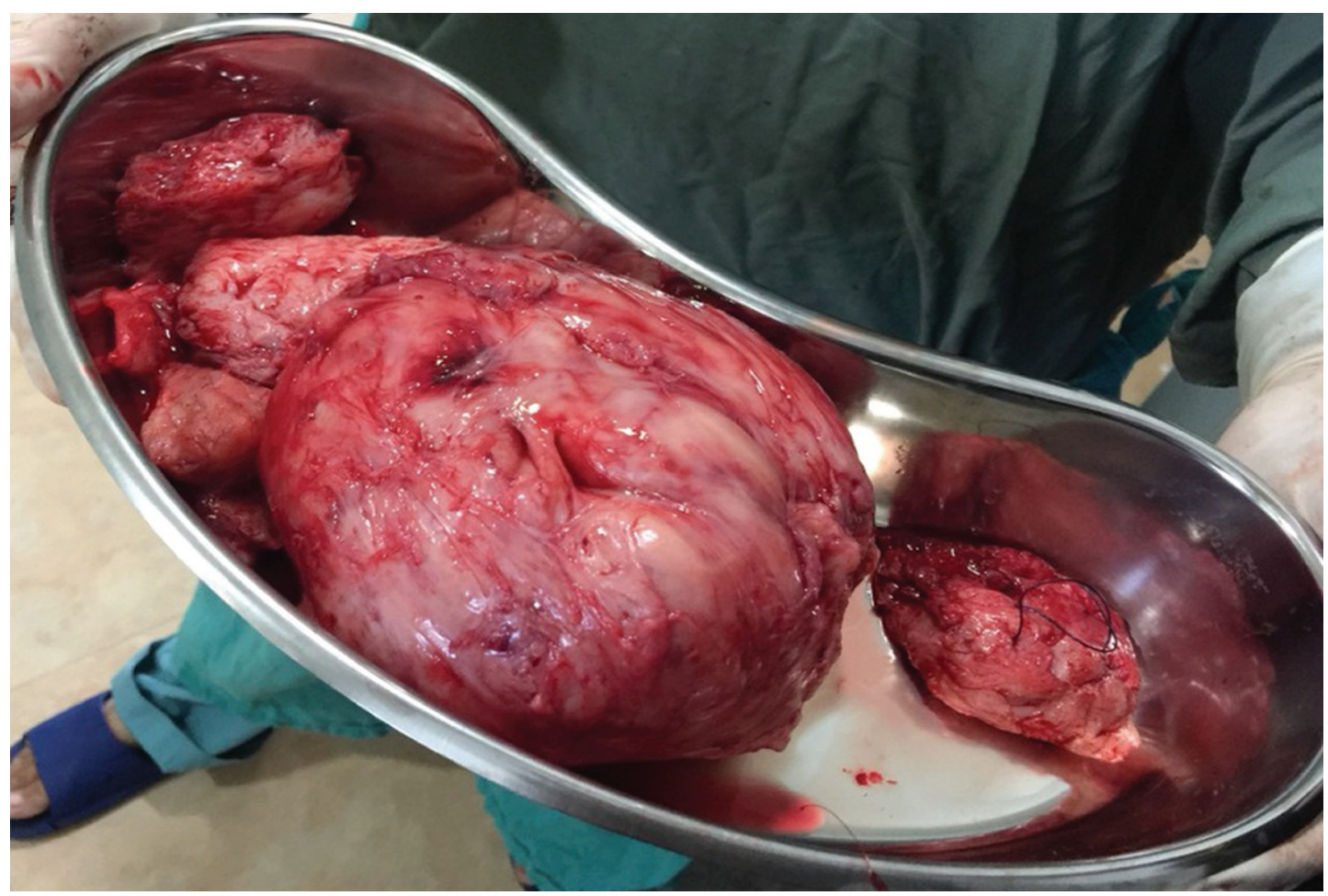

Figure 1: The excised mass on gross examination revealed grey white nodular tissue measuring $13.5 \times 8 \times 2.5 \mathrm{~cm}$. Cut section showed grey white areas with whorled appearance.

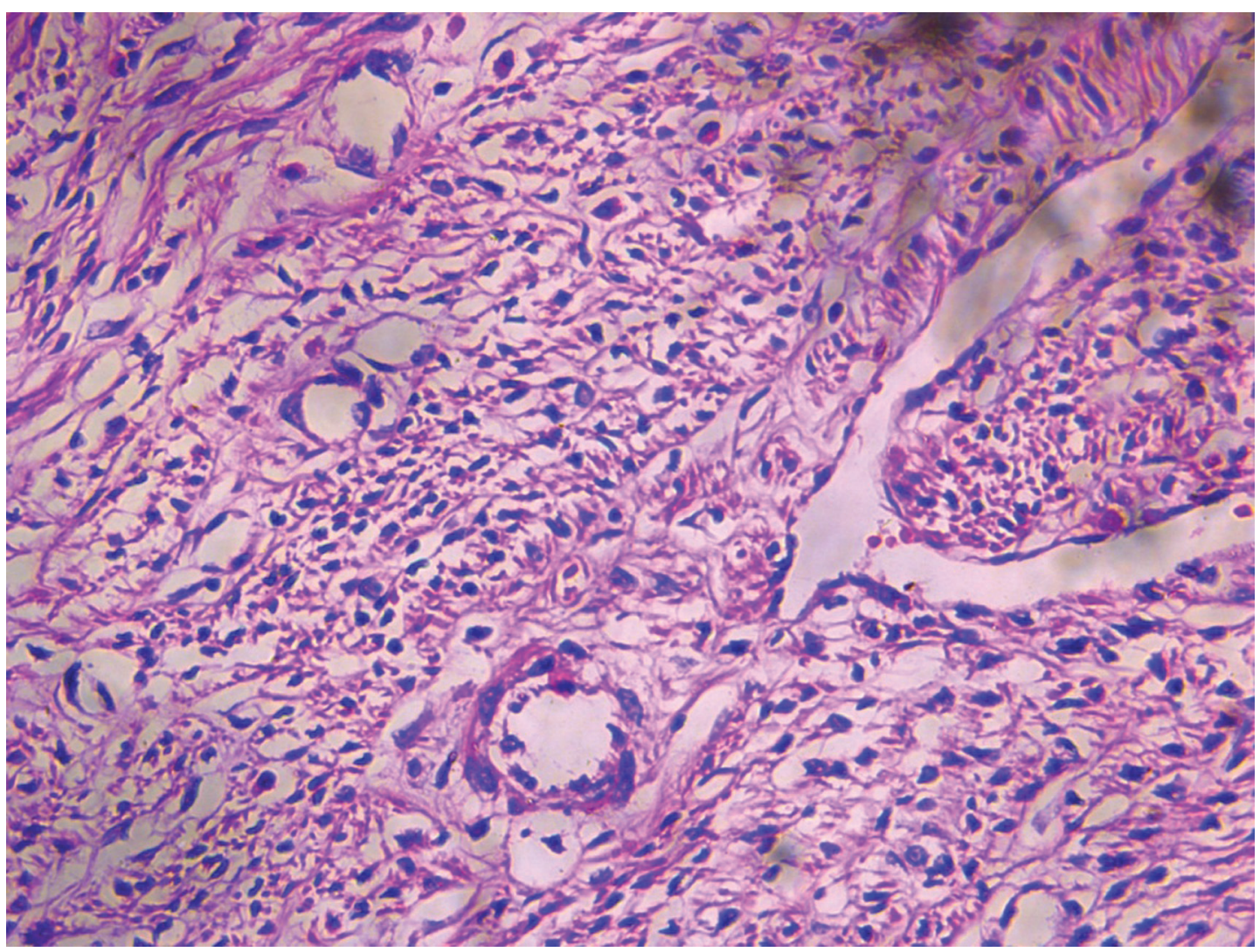

Figure 2: Microscopic examination: Interlacing bundles of smooth muscles intersecting each other. Sections from the separate tissue shows muscle fascicles which were separated by abundant extracellular mucin producing a striking myxoid appearance (myxoidosis). 
neurofibromatosis. Mccluggage et al. ${ }^{1}$ reported another 3 patients as an unusual peudoneoplastic condition of uncertain etiology. One of the 3 patients of latter study had neurofibromatosis and was included in the study by Pugh et al $^{3}$.

In our case, we did not find any connective tissue disease. Similar to the case reported by Veras et al., our patient also had enlarged uterus with myometrial thickening (secondary myometrial hypertrophy). Histologic finding of our case was similar to as described by the author ${ }^{2}$. But unlike their case, we also encountered a huge leiomyoma. In the study by Mccluggage et al. immunohistochemistry revealed positive CD34 and CD10 which was not seen in our case. Occasional myxomas have been reported in the uterus, including in a patient with Carney's syndrome ${ }^{4}$. Myxoid change may also occur in the stromal component of carcinosarcomas, adenosarcomas, and undifferentiated sarcomas, and also in undifferentiated carcinomas ${ }^{5}$. Marked edema may mimic myxoid stromal change, like in uterine smooth muscle tumors may exhibit pronounced edema resulting in a nodular appearance, socalled perinodular hydropic degeneration, and this can be mistaken for myxoid change ${ }^{6}$. Apart from myxoid leiomyosarcoma, myxoid endometrial stroma sarcoma and inflammatory myofibroblastic tumor, a common non neoplastic condition that should be included in differential diagnosis is pregnancy ${ }^{2}$. Third trimester uterus after hysterectomy for various obstetric complications show enlarged uterus with myometrial thickening and histologically myometrial smooth muscle fascicle are separated from one another due to interstitial edema ${ }^{6}$.

\section{Conclusion}

Though a rare occurrence, myometrial myxoidosis should be considered in patients with uterine mass and infertility. Most of myometrial myxoidosis cases are diagnosed after histopathological examination and they should be evaluated for lupus erythematosus.

\section{References}

1. McCluggage WG, Young RH. Myxoid change of the myometrium and cervical stroma: description of a hitherto unreported non-neoplastic phenomenon with discussion of myxoid uterine lesions. Int J Gynecol Pathol 2010;29:351-7.

2. Veras E, Junkins-Hopkins JM, Marinis S, et al. Myometrial myxoidosis: a report of 2 cases of a distinctive type of secondary myometrial hypertrophy in patients with lupus erythematosus. Int J Gynecol Pathol 2009;28:164-71.

3. Pugh A, McCluggage WG, Hirschowitz L. Multifocal Uterine Myxoid Change: A newly recognized association with Neurofibromatosis Type 1. Int J Gynecol Pathol 2012;31: 580-3

4. Barlow JF, bu-Gazeleh S, Tam GE, et al. Myxoid tumor of the uterus and right atrial myxomas. S D J Med 1983;36:9-13.

5. Silva EG, Deavers MT, Bodurka DC, et al. Association of low-grade endometrioid carcinoma of the uterus and ovary with undifferentiated carcinoma: a new type of dedifferentiated carcinoma? Int J Gynecol Pathol 2006;25: 52-8.

6. Clement PB, Young RH, Scully RE. Diffuse, perinodular, and other patterns of hydropic degeneration within and adjacent to uterine leiomyomas: problems in differential diagnosis. Am J Surg Pathol 1992;16:26-32. 